\title{
The Synthesis of Large Molecules*
}

\author{
By Prof. H. Mark, University of Vienna
}

$\mathrm{N}^{\circ}$

RMAL molecules with which the organic chemist has been concerned for many years, and which have led to an enormous number of interesting and important chemical combinations with valuable properties, have molecular weights between 50 and 2,000. Only a very few of them are smaller and few are larger, but even then they do not exceed these limits by a considerable amount.

For some time, it has been known that certain types of chemical reactions lead to products which do not crystallize and to which therefore was attributed the name of 'resins' or 'resin-like' substances. To identify and reproduce them was very difficult; hence for a long time it was considered that they could not be taken as objects for scientific investigation. But these substances offered mechanical, thermal and electrical qualities which made them very important from the technical point of view, and interest in their structure and synthesis began to develop.

On the other hand, the study of such natural products as cellulose, proteins, rubber, starch, silk, chitin, etc., led to the conclusion that their structural principles were the same as those of the synthetic resins; and the interest in this group of bodies was intense in view of their biochemical and technical qualities. Therefore a number of scientific workers began to investigate large molecules with enthusiasm; and our knowledge of them has rapidly increased.

It was found that two types of large molecules exist, one with chain-like molecules, another with large molecules which have the structure of a twoor three-dimensional network. In both cases, the number of atoms which are held together by normal chemical main valencies is very largebetween $10^{3}$ and $10^{8}$-and hence their molecular weight lies between $10^{4}$ and $10^{8}(O=16)$. Several other qualities of these large molecules have been studied and cleared up; for example, their shape, reactivity, mechanical and optical behaviour, ete.

After our knowledge on the outstanding qualities of these bodies had reached a certain point, interest was aroused in that type of chemical reaction by which such macro-molecules are built up, and it is proposed to give a very short survey of the present state of evidence of this kind of chemical reactions, the so-called polymerization and polycondensation reactions.

- Friday evening discourse delivered at the Royal Institution on April 23.

\section{The Polymerization Reaction}

Small organic molecules with one or more double bonds have the peculiarity of polymerizing under certain conditions of temperature, pressure, etc. This polymerization leads mostly to macromolecular substances, which are therefore also often called high polymers or high polymeric substances. Let us consider the most simple chemical molecule with a double bond, namely, ethylene and its derivatives.

In this case the total polymerization reaction (of an ethylene derivative) is given by the relation

$$
n . \mathrm{CH}_{2}=\mathrm{CHX} \rightarrow\left(\mathrm{CH}_{2} . \mathrm{CHX}\right)_{n} .
$$

In this equation, $X$ signifies the substituent $\left(\mathrm{H}, \mathrm{CH}_{3}, \mathrm{Cl}_{1} \mathrm{C}_{6} \mathrm{H}_{5}, \mathrm{OH}\right.$, etc. $), n$ is a number of the order of magnitude between $10^{2}$ and $10^{4}$. The left side of the equation (1) represents a great number of independent small molecules, each of which is completely known regarding its structure and contains a double C-C linkage. The right side gives a large molecule, the structure of which is not quite clear in all its details, which contains all the atoms contributed by the $n$ molecules linked together by main valence bonds.

Frequently the macro-molecules built up from ethylene monomers are chains, and in such cases one may write equation (1) in more detail thus:

$$
\begin{aligned}
& n . \mathrm{CH}_{2}=\mathrm{CHX}- \\
& \mathrm{CH}_{3}-\mathrm{CHX}-\left(\mathrm{CH}_{2}-\mathrm{CHX}\right)_{n-2}-\mathrm{CH}=\mathrm{CHX} .
\end{aligned}
$$

In this case, a certain assumption is made as to the internal structure of the chain-like macromolecule, an assumption which is justified in some cases by considerable experimental evidence. Many chain polymerization processes were carried out with substances of this kind and formulated by relations of type (2), and it may be pointed out that Staudinger in Germany and Carothers in the United States especially have worked systematically in preparing macro-molecules by the aid of such processes.

Besides the material which is built up hereby, and which at first absorbed the whole attention of investigators, the mechanism of the process by which such long chains are formed is also of great interest and hence should be studied systematically. We have therefore in Vienna in the last five years earried out some series of experiments to elucidate 
the different steps of typical polymerization reactions.

From the beginning, it is clear that the numerous independent particles on the left side of equation (1) cannot be linked together in one single collision process, which would have a vanishing probability, but that there must be a kind of growth, which builds up the long chain. This growth process is the aim of our studies; it seems to be not only of scientific interest in connexion with the physicalchemical problem of reactivity, but also of technical and biochemical importance. The technical significance may be confirmed by the fact that a great number of synthetic high polymers are of considerable interest, such as artificial rubber, insulators, varnishes, etc., while the biochemical importanc $\ni$ is indicated by the observation that our own bodies and the skeletal substances of all plants and animals are built up of high polymeric substances and that therefore the growth processes of organized Nature may be connected with the reactions studied. In any event, a profound knowledge of the simplest polymerization reactions will be indispensable for the understanding of natural growth. We thought, therefore, that it might be worth while to devote some work to a thorough investigation of the kinetics of polymerization.

The general situation was not unfavourable: the kinetics of normal chemical reactions even of a rather complicated type was cleared up recently to a considerable extent by such scientific workers as Abel, Bodenstein, Bonhoeffer, Hinshelwood, Polanyi, Rideal, Semenoff, H. S. Taylor and others. The experimental procedure is not too complicated and the methods of following gradually the rate of the process are more or less worked out. Besides our own studies (carried out in the Chemical Institute of the University in Vienna by Breitenbach, Dostal, Jorde, Marecek, Pilch, Raff, Rudorfer and Suess) in the last four or five years, authors such as Bawn, Chalmers, Flory, Melville, Norrish, Rideal, Schulz, Taylor, Vernon and others have contributed valuable results, and one can summarize the present state of our knowledge in the following way.

(1) The whole polymerization process is very complicated, even when only chains are built up, and depends to a high degree on such experimental conditions as temperature, pressure, solvent, presence of catalytically active bodies, etc. But in the simpler cases-and only these have been studied carefully up to date-one can always distinguish three typical steps in a reaction of this kind.

(a) The building of germs or nuclei. No polymerization can take place if there is not an initiation process, by which unsaturated and highly reactive 'germs' are formed. This process can be monomolecular, bimolecular or of another order, and furnishes the centres of growth. It consists in the fact that in a certain molecule a very high amount of activation energy or unsaturation is concentrated either by impact with another particle of high energy, or by absorption of a photon or by the formation of an intermediate combination. In any event, this germ-building reaction is rather slow and very dependent on the temperature or on the presence of a catalyst (including light).

This first step has been called the 'starting' process, and we can formulate a thermal bimolecular starting reaction, for example,

$$
\begin{aligned}
\mathrm{CH}_{2}=\mathrm{CHX}+ & +\mathrm{CH}_{2}=\mathrm{CHX} \rightarrow \\
& -\mathrm{CH}_{2}-\mathrm{CHX}-\mathrm{CH}_{2}-\mathrm{CHX}-,
\end{aligned}
$$

where the nucleus on the right side has two free chemical main valencies and therefore represents a very high free energy. Consistent observations by different workers led to the conclusion that the activation energy of the starting reaction in the case of polymerization of ethylene derivatives lies between 20,000 and 30,000 cal. per mol. The reaction is therefore slow and the germs are produced with a limited velocity.

(b) The reaction of growth. If an unsaturated germ is formed in the solution, there are two possibilities for its further fate. It may collide with another molecule (monomeric substance or solvent) and be desactivated by the impact. In this case, the nucleus just produced is annihilated again and no polymerization is started. But the germ may also react with the colliding particle and, if it is a monomeric molecule, may add it according to the relation :

$$
\begin{gathered}
-\mathrm{CH}_{2}-\mathrm{CHX}-\mathrm{CH}_{2}-\mathrm{CHX}-+\mathrm{CH}_{2}=\mathrm{CHX} \rightarrow \\
\quad-\mathrm{CH}_{2} \ldots \mathrm{CHX}-
\end{gathered}
$$

This is a 'growth step' and processes of this type lead to the result required, namely, to long chains, supposing that they follow one another very quickly. Really high polymeric bodies can only be formed when a slow germ production is followed by a quick growth reaction.

In fact the rate of reaction $(b)$ is very much higher than that of $(a)$. Measurement shows that (b) goes at least $10^{4}$-fold quicker than $(a)$; every nucleus is built, begins at once to grow, and rapidly gives a chain of considerable length. This high velocity is easily understood: the free valencies at the end of the germ and of all inter. mediate members of the growing chain represent a high energy, and therefore the activation energy of the addition reaction $(b)$ is rather low, lying between 4,000 and 8,000 cal. per mol., and explains the rapid growth, which is nearly independent of 
temperature. Very interesting results concerned with the steric factor of growth would lead us much too far and cannot be considered here.

If the growing chains meet no obstacle during their period of adding monomolecular material, they will grow so long as molecules of the polymerizing substance are available, and the result of the whole process will depend only on the velocity of germ formation, $k_{1}$, and of growth rate, $k_{2}$. The competition of these two influences was discussed quantitatively by Dostal and Mark, and formulæ have been derived and compared with the experimental results. It seems that only in very special cases one can reduce the whole complicated polymerization process to these two steps alone; mostly it is necessary to take into account a third elementary effect, which interferes with these two steps, namely, the breaking-off of the growth.

(c) Breaking-off processes and sudden finishing of growth. Our general knowledge of addition reactions between molecules shows that with in. creasing size of the reacting particles the probability for a successful collision, that is, the addition of a new member in the chain, decreases very rapidly with increased size of the molecules, even if the activation energy remains quite constant. Investigations of Evans, Eyring, Hellmann, Polanyi, Syrkin and Wassermann show that the reactivity falls off very quickly even with medium molecular weight $(200-400)$. It is therefore to be expected that the rate of growth would be very much dependent on the actual chain length, an influence which was introduced into the formulæ by Dostal and Mark, with the consequence that the growth-rate of an individual chain falls off slowly. Comparison with experiment showed that under certain conditions the polymerization of styrene seems to be remarkably influenced by this peculiar effect.

On the other hand, one must be aware of the fact that a third type of reaction finishes the growth of the chains by annihilating the active groups at their ends. Thus we have to introduce a third reaction constant, $k_{3}$, which summarizes all possibilities for the saturation of the reactive spots. They are rather numerous, and it is therefore difficult to get a clear idea of the chain-breaking process.

(1) When the chains are flexible, it may happen that the two free valencies on their ends react with one another producing a ring molecule of many members. Such molecules are known from the work of Müller, Ruzicka and others, and even the kinetics of their formation was cleared up to a certain degree by Freundlich and Salomon. We do not yet know exactly how much this factor influences a given polymerization reaction, because we have no effective means of finding out how many ring molecules are present in the product of a particular polymerization reaction. In this connexion, new experimental evidence is wanted ; such a breaking-off reaction would be monomolecular.

(2) A growing system can loose its active ends, if a hydrogen atom wanders along the chain and if a double bond is formed at one end :

$$
\begin{array}{r}
-\mathrm{CH}_{2}-\mathrm{CHX}-\ldots-\mathrm{CH}_{2}-\mathrm{CHX}-\underset{\mathrm{CH}}{-}-\mathrm{CHX}-\ldots-\mathrm{CH}=\mathrm{CHX}
\end{array}
$$

The probability of such isomerization will be inversely proportional to the actual length of the chain, and a breaking-off reaction of this type will be of the first order, short chains being in greater danger of undergoing this isomerization than longer ones. Actually, in the case of short chains $(n=2$ or 3$)$, the final products of this reaction could be isolated in one or two cases, but it is not yet clear what role this type of chain-cutting plays in the whole process.

(3) The annihilation of a single active group at one end of the growing thread-like molecule can also be accomplished during a collision according to the equation

$$
\begin{array}{r}
-\mathrm{CH}_{2} \ldots \mathrm{CHH}^{*}-\mathrm{CHX}-+M \rightarrow \\
-\mathrm{CH}_{2} \ldots \mathrm{CH}=\mathrm{CHX}+M \mathrm{H}^{*}
\end{array}
$$

Here the hydrogen atom $\mathrm{H}^{*}$ jumps under the influence of the collision with $M$ from the penultimate carbon atom to the colliding particle $M$, and a double bond is formed. Several types of this reaction have been discussed by Dostal, Flory, Mark and Schulz. The probability of such a process is proportional to the concentration of the unsaturated ends and to the concentration of $M$. The latter partner may be any molecule able to take up a hydrogen atom present in the reacting system, for example, a normal monomeric particle, another growing chain, a solvent molecule or any impurity of the solution. Even the wall of the vessel could produce the change expressed by the relation $(d)$. Therefore it is not easy in a given case to say precisely what will happen.

An important and interesting question is : What happens to the energy which is set free by the process $(d)$ ? As a new C-C bond is formed, we may estimate the energy excess of $(d)$ to be about 70,000 cal. per mol. It may remain in the chain and increase its internal vibrations and rotations; perhaps with the effect that after a certain average life-time the second end of the chain is also stabilized by a similar action. But the energy may also be transferred to $M$ and create a highly active particle. If $M$ is a monomeric molecule, it can be transmutated into a new germ, so that one chain is ended by $(d)$ but another is started. There are signs that something like that happens under 
certain experimental conditions. If $M$ is a solvent molecule, the energy may be dissipated by consecutive collisions, but it may also be attached to one monomeric particle in the course of reproduction of a germ with the aid of the solvent. In recent papers of Dostal, Flory, Mark, Schulz and others, these various possibilities are discussed and brought into relation with the experimental facts.

The result is that at present no polymerization process can yet be resolved quite clearly into all its elementary steps, but there is no doubt that the three above-mentioned processes, namely, chain start, chain growth, and chain ending always take the outstanding role during a polymerization reaction. Increasing experimental material will enable us to separate more and more neatly the different possible steps and to work out something like a fine structure of polymerization reactions.

\section{Polycondensation Reactions}

This is the second type of reaction by which high polymers can be produced. Here also we will confine ourselves to reactions which lead to pure chain polymers. In a condensation reaction a new molecule is built by removal of a part of the reactant particles; this part is usually water. If a dibasic acid, for example, succinic acid, reacts with glycol one gets :

$$
\begin{gathered}
\mathrm{HO} \cdot \mathrm{H}_{2} \mathrm{C}-\mathrm{CH}_{2} \cdot \mathrm{OH}+\mathrm{HOOC} \cdot \mathrm{CH}_{2} \cdot \mathrm{CH}_{2} \cdot \mathrm{COOH} \rightarrow \\
\mathrm{HOH}_{2} \mathrm{C} \cdot \mathrm{CH}_{2} \mathrm{O} \cdot \mathrm{OC} \cdot \mathrm{CH}_{2} \cdot \mathrm{CH}_{2} \cdot \mathrm{COOH}+\mathrm{H}_{2} \mathrm{O} \quad(e)
\end{gathered}
$$

This esterification leads to a molecule which can again react with an alcohol or with an acid, producing further reactive molecules and growing thus slowly to a long chain with alternate alcohol and acid members.

Reastions like this have been investigated recently by Dostal, Flory, Marecek, Raff and others. They are much easier to deal with because the intermediate products are not unsaturated but of the same type as the monomeric substance. It is therefore not a chain reaction, which we have before us, but a stepwise esterification for which kinetic formulæ can be derived without serious difficulties. The problem of the mutual interaction of three different elementary processes as it has to be solved in the case of polymerization is here reduced to the mathematical study of a step reaction with many steps. The production of 'germs' is here of the same order of magnitude as the velocity of growth, and therefore the chains which are formed are much shorter than in the case of polymerization. Therefore a fairly good agreement was obtained by comparing the results of experiments with the above-mentioned formulæ, and the problem of polycondensation reactions does not offer serious difficulties to a quantitative understanding.

This is a reason why the efforts of workers are being more and more concentrated on the study of polymerization processes, which still offer a great number of unsolved and interesting problems.

\section{The Solar Eclipse of June 19, 1936}

A $\mathrm{N}$ old practice was revived on May 27, when a joint meeting of the Royal Society and the Royal Astronomical Society was held to receive reports on the expeditions organized by the Joint Permanent Eclipse Committee of the two societies for the total solar eclipse of June 19, 1936. It was sad to observe that on this occasion the tradition by which the president of the Royal Astronomical Society sat facing the audience alongside the president of the Royal Society was allowed to lapse.

Prof. F. J. M. Stratton opened the discussion with an account of the expedition to Kamishari in Hokkaido, staffed from the Solar Physics Observatory, Cambridge, with the addition of Dr. T. Royds, of the Kodaikanal Observatory, sent by the Government of India. $\mathrm{He}$ mentioned that the site was chosen after consultation with the Meteorological Office and consideration of the data supplied by the National Research Council of
Japan. Unfortunately, the prevailing wind which should have given the desired weather for the eclipse chose the wrong week to prevail, and the day of the eclipse was cloudy. The second half of the partial phase was happily clear, and Dr. Royds was enabled to carry through most of his programme successfully; second contact was observed through gathering elouds which spoilt by scattering light the programme of observing intensities of chromospheric spectral lines at different heights above the sun's limb, though flash spectra were obtained; within five seconds of the commencement of totality the sun was completely covered by cloud too thick even for the infra-red cameras to penetrate; the sun did not emerge into a clear sky until 10 minutes after the end of totality.

It was unfortunate that the half of the eclipsed sun which was in clear sky for second contact and for the first few seconds of totality was on the 